\title{
Cerebral correlates of cognitive aging: Gray-white-matter differentiation in the medial temporal lobes, and fluid versus crystallized abilities
}

\author{
NAFTALI RAZ \\ Memphis State University, Memphis, Tennessee \\ DARYL MILLMAN \\ Chicago Medical School, North Chicago, Illinois \\ and \\ GÜNSELI SARPEL \\ North Chicago VA Medical Center, North Chicago, Illinois
}

\begin{abstract}
We investigated the relationship between age, structural properties of selected cerebral regions, and cognitive performance in healthy adults, 18 to 78 years old. Spin-lattice relaxation time (T1), measured by nuclear magnetic resonance, was used to describe the structural composition of the brain tissue. Temporal lobe white-matter $\mathrm{T} 1$ showed age-related prolongation best described by a quadratic polynomial. There was a significant cubic trend in the association of hippocampal (gray-matter) T1 with age. In the examined regions of the medial temporal lobes, normally observed differentiation between gray- and white-matter $\mathrm{T} 1$ diminished linearly with age and disappeared almost completely in the elderly. Age and the ratio of gray- to white-matter T1 accounted for $53 \%$ of the variance in a measure of fluid intelligence (Cattell Culture Fair Test); the unique contributions of age and of gray-white-matter $\mathrm{T} 1$ ratio were $23 \%$ and $3 \%$, respectively. The largest share of the variance in fluid intelligence $(27 \%)$ was explained by the common influence of age and gray-white-matter T1 ratio. The same set of variables explained no significant proportion of the variance in crystallized intelligence. The possible mechanisms underlying age-related changes in gray-white-matter differentiation, their relationship to age-related selective deterioration of cognitive functions, and the implications of the findings for research on biological markers of aging are discussed.
\end{abstract}

Aging is associated with specific changes in cerebral morphology, as well as with differential decline in cognitive abilities (Coleman \& Flood, 1987; Horn, 1986). Cumulative evidence from postmortem studies suggests that the regions of the brain that are more likely to undergo age-associated alterations are the medial temporal lobes encompassing the hippocampal formation and the prefrontal cortex (Barnes, 1983; de Leon, George, Stylopoulos, Smith, \& Miller, 1989; Mani, Lohr, \& Jeste, 1986; Terry, DeTeresa, \& Hansen, 1987). In the cognitive domain, a group of abilities such as nonverbal reasoning, rule discovery, and concept formation, usually called fluid intelligence, is especially vulnerable to the effects

\footnotetext{
We thank W. Hindo, Radiology Service, North Chicago VA Medical Center, for his support. We gratefully acknowledge the assistance of the MRI technicians: D. King, S. Damhoff, K. Krueger, and S. Tarr. The comments of Sarah Raz and two anonymous reviewers are greatly appreciated. This research was supported in part by the Biomedical Research Support Grant S07-RR0-5366-26 to N. Raz through the Chicago Medical School.
}

of aging. On the other hand, formal verbal reasoning, comprehension of culture-specific rules and strategies, and general fund of knowledge-the abilities constituting crystallized intelligence-do not decline with age (Horn, 1986).

Pathological changes in the temporal lobes, mainly in the hippocampus, have been implicated in cognitive declines observed in the normal elderly and are regarded as one of the major signs of age-related cognitive pathology (Squire, 1986; Winocur, 1988). Horn (1986) has suggested that cognitive components constituting fluid intelligence can be affected by lesions in the medial temporal and limbic structures. He also reported age-related decrease of regional cerebral blood flow (rCBF) in broadly defined medial temporal aspects of the brain. A relationship between the extent of hippocampal atrophy and memory deficit has been demonstrated in several cases of traumatic amnesia (Press, Amaral, \& Squire, 1989).

Until recently, a direct test of any hypothesis relating differential decline of cognitive abilities to age-related deterioration of cerebral structures has been impossible due 
to the lack of instruments permitting an in vivo assessment of neuroanatomy and brain function in healthy individuals. Introduction of computerized imaging techniques-computer-assisted tomography (CT) and magnetic resonance imaging (MRI)-has created new opportunities for evaluation of age-related neuroanatomical changes in intact humans. In addition to allowing visualization of brain structures in any anatomical plane, MRI provides an opportunity for an in vivo evaluation of the molecular behavior of water, the main constituent of cerebral tissue (Harms \& Kramer, 1985).

Contemporary MRI scanners provide two basic parameters describing the behavior of water protons in living tissue: spin-lattice (longitudinal) relaxation time (T1) and spin-spin (transverse) relaxation time (T2). Both $\mathrm{T} 1$ and $\mathrm{T} 2$ are sensitive to differences in molecular organization of healthy and diseased tissue (Bottomley, Foster, Argersinger, \& Pfeifer, 1984). Although for a given magnetic field strength $\mathrm{T} 1$ correlates highly with water content of the brain $(r>.9)$, it is influenced by changes in the macromolecular structure of water as well (Bottomley et al., 1984; MacDonald et al., 1985; Unger, Littlefield, \& Gado, 1988). The dependence of $\mathrm{T} 1$ on the relative share of bulk-phase and hydration-layer water makes it a sensitive tissue-typing tool (Mathur-DeVré, 1984). In general, tissue containing a substantial amount of bulk water has longer $\mathrm{T} 1$ than tissue in which water is represented, mainly in hydration layer clinging to macromolecules (Mathur-DeVré, 1984; Unger et al., 1988). As a result, cerebral gray matter would normally have considerably longer $\mathrm{T} 1$ time constants than the white matter.

Water content of the white matter increases dramatically with age (Wiggins et al., 1988). Focal edema due to microinfarctions (Tomonaga, Yamanouchi, Tohgi, \& Kameyama, 1982), atrophic perivascular demyelination (Ansari \& Loch, 1975), cumulative hypoxic-ischemic damage (Ginsberg, Hedley-Whyte, \& Richardson, 1975), and loss of proteins and glycolipids from the myelin sheath (Kirkpatrick \& Hayman, 1987; Wiggins et al., 1988) are associated with aging and may produce molecular changes causing prolongation of $\mathrm{T} 1$ in the white matter. Indeed, perivascular demyelination has been histopathologically confirmed in many cases of the white-matter lesions detected with MRI (Kirkpatrick \& Hayman, 1987). Wahlund et al. (1990) reported that age-related changes in white-matter composition are reflected in altered $\mathrm{T} 1$ values. In their study, conducted on an age-restricted sample of elderly subjects, the correlation between age and frontal lobe white-matter $\mathrm{T} 1$ averaged across the hemispheres was $r=.60$. In a recent study of a representative sample of normal volunteers, Jernigan et al. (in press) reported a multiple correlation of $r=.68$ between a quadratic function of age and white-matter $\mathrm{T} 2$.

The $\mathrm{T} 1$ values of the gray matter may also be affected by a variety of age-related processes. Postmortem studies indicate that aging is accompanied by a decrease in neu- ronal size and density, formation of neurofibrillary tangles and plaques, emergence of Hirano bodies, granulo-vacuolar degeneration, accumulation of lipofuscin, and proliferation of glia (Bourne, 1973; Coleman \& Flood, 1987; Terry et al., 1987; Tomlinson, Blessed, \& Roth, 1968; Wisniewski \& Terry, 1973). The net result of these processes, associated with normal aging as well as with age-related pathology, is introduction of dense structures into the gray matter. These changes are likely to decrease the share of bulk water and subsequently shorten gray-matter relaxation times. On the other hand, atrophic cell loss and subclinical infarctions may produce increases in the bulk water content of the gray matter and prolong $\mathrm{T} 1$.

The interaction among the described changes in gray and white matter is complex. While the age-related processes in the white matter lead to consistent prolongation of $\mathrm{T} 1$, the pathophysiological changes in the gray matter may result in either prolongation or shortening of the spin-lattice relaxation times. Consistent prolongation of the T1 times in the white matter would result in reduced differentiation between the two types of tissue even if relatively stable gray-matter $\mathrm{Tl}$ values were maintained. Should factors causing shortening of gray-matter T1 prevail, the difference between the MRI appearance of white and gray matter would virtually disappear. At least one report suggests an age-related reduction of apparent gray-white-matter discriminability on CT scans (George, de Leon, Ferris, \& Kricheff, 1981). This phenomenon has not been assessed quantitatively with modern neuroimaging techniques. In light of George et al.'s (1981) report, direct measures of $T 1$ values in gray and white matter would be expected to show age-related decrease in gray-white-matter differentiation.

In this study we employed a sensitive and reliable method of direct on-line computation of T1 (Morone et al., 1987). Although this method is well suited for assessment of regional properties of brain tissue, it cannot be applied to a large number of brain regions at once. Thus, the measurements were conducted in the areas of the brain that have been implicated in age-related changes in cognitive functions, that is, the medial temporal lobes incorporating the hippocampal formation. In light of the evidence supporting the role of temporal lobe structures in age-related cognitive changes, we hoped that choosing the medial temporal lobes for T1 measures would increase our chances of finding cerebral markers of cognitive aging.

\section{METHOD}

\section{Subjects}

The sample consisted of 26 healthy volunteers who reported no history of psychiatric or neurological disorders, alcoholism, diabetes, or hypertension-all of which are known to produce neuromorphological changes and to disrupt cognitive functioning of the elderly (White, Cartwright, Cornoni-Huntley, \& Brock, 1986). All elderly subjects lived independently, and some were still gainfully 
employed. All subjects scored at least 29 out of 30 points on the Mini-Mental Status Examination (MMSE; Folstein, Folstein, \& McHugh, 1975). The mean age in this sample, which included 15 males and 11 females, was 45.8 years $(S D=21.8)$, with a range of 18 to 78 years. The average duration of formal education was 14.7 years; the correlation between age and education was $r=$ -.29 , n.s. No formal assessment of handedness was conducted, but all subjects were self-reported right-handers. They signed an informed consent form and were thoroughly briefed regarding the objectives of the study.

\section{Apparatus and Procedure}

Imaging and spin-lattice relaxation time (T1) measurements were performed on a Fonar Beta-3000 0.3T permanent-magnet scanner at the McCormick University Clinics (University of Health Sciences/Chicago Medical school). All imaging was conducted in a spin-echo (SE) mode with a $256 \times 256$ matrix and a $22-\mathrm{cm}$ field of view. A $27-\mathrm{cm}$ head coil was used in all procedures.

Before the T1 measures were taken, two imaging sequences were performed. Nine sagittal slices were obtained at echo time (TE)/repetition time (TR) $=28 / 600 \mathrm{msec}$, with three averages per projection and slice thickness/interval of $4.2 / 6.0 \mathrm{~mm}$. Upon completion of the 7-min sagittal sequence, a coronal sequence was initiated. In that sequence, 17 to 21 slices (depending on the cranial size) were taken in the coronal plane, with TE/TR $=28 / 1,800 \mathrm{msec}$, two averages per projection, and slice thickness/interval of $6.6 / 8.6 \mathrm{~mm}$. The midsagittal slice was used as a scout, and the position of the coronal slices was determined in reference to the middie of the pituitary gland. Acquisition of the coronal sequence took about $15 \mathrm{~min}$.

All scans were examined by a neurologist with extensive experience in clinical MRI (G.S.). One subject (76-year-old female) showed moderate parietal atrophy, and 2 subjects (71-year-old male
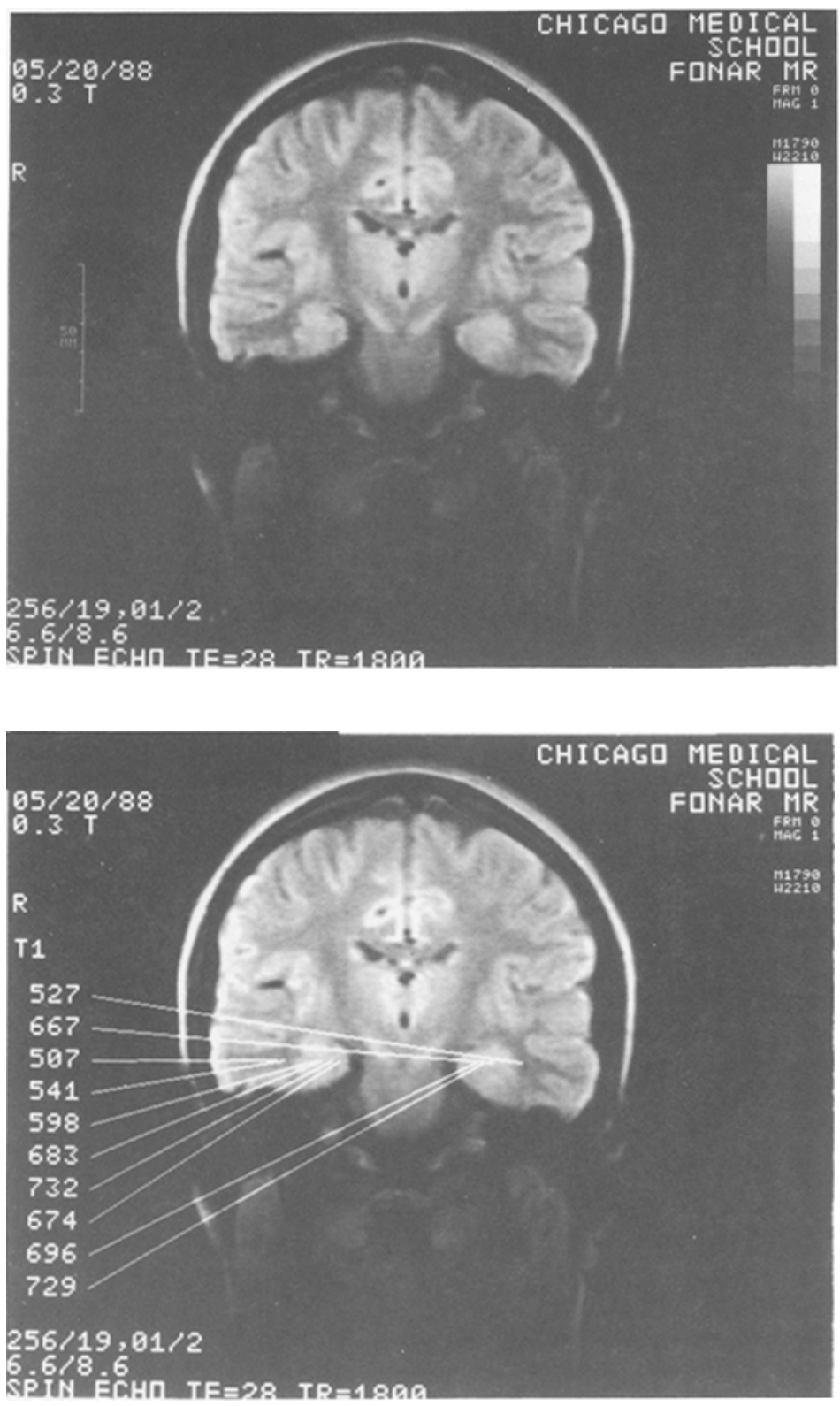

Figure 1. Top: A coronal MRI slice through anterior hippocampal formation used for T1 measurement. Bottom: The same slice with superimposed T1 values. 
and 68-year-old female) had minor periventricular patches of increased signal intensity. Otherwise, no space-occupying lesions or significant areas of signal hyperintensity were observed, and scan appearance was judged normal for age. As indicated by the MMSE scores and the interview, the subjects with positive neuroradiological findings exhibited neither clinical-behavioral harbingers of mental deterioration nor a pattern of risk factors characteristic of dementing illness. The finding of areas of hyperintensity per se is not indicative of pathology (Leys et al., 1990; Sze et al., 1986) and does not necessitate exclusion of the subjects.

\section{Measurement of T1}

The measurements were conducted on four regions of interest (ROI) on a single slice. After the imaging sequences were completed, the subject remained in his/her position, and a coronal slice (Figure 1, top) was selected to obtain the T1 values. That slicethird caudally from the middle of the pituitary-was selected because it allowed a clear view of the hippocampal formation. The selection was verified against the atlas of correlative MRI neuroanatomy (Daniels, Naughton, \& Naidich, 1987). Although the investigators who performed $\mathrm{T} 1$ measurement were not blind to the subjects' age, the use of clearly defined neuroanatomic landmarks and uniform slice selection procedures made bias in selection of the ROI rather unlikely.

The T1 values were computed for the anterior part of the hippocampal formation and the adjacent subcortical white matter in both hemispheres. The computations were performed on-line using a strip method developed by the Fonar Corporation, Melville, NY, and described by Morone et al. (1987). In this method, the acquisition of $T 1$ is separated from the imaging process. It is performed with an SE pulse sequence and time-varying gradients at 13 different repetition rates. At the shortest repetition time (TR), signal intensity is the greatest, dropping exponentially with prolongation of the TR. The T1 measurement is based on the linear regression of the logarithm of relative decrement in signal amplitude on the repetition time. In comparison with conventional methods, in which T1 is computed off-line and is based on only two TR values, the strip method is distinguished by relatively high reliability. Its main drawback is that the measurement is restricted to one relatively small region at a time.

Thirteen TR values were used in each T1 strip computation, and the total acquisition time was $3.5 \mathrm{~min}$. The spatial resolution of the T1 measurement was $7 \mathrm{~mm}$, and, for each strip, T1 values from up to 10 points along the strip were recorded. An example of a strip with $10 \mathrm{~T} 1$ values displayed is presented in the bottom panel of Figure 1. The T1 measurement sequence was repeated until at least six measurements per ROI were obtained. The median TI value for a given ROI served as its representative $\mathrm{T} 1$.

\section{Psychometric Tests}

To assess cognitive functions, two tests, one representative of fluid and one of crystallized intelligence, were used. The Cattell Culture Fair Test (CFIT; Cattell \& Cattell, 1973) and Extended Vocabulary (V3) from Educational Testing Services FactorReferenced Test Kit (Ekstrom, French, Harman, \& Derman, 1976) were administered to each subject individually in a span of no more than a week from the date of MRI scanning. The total number of correct responses on CFIT was used as a measure of fluid intelligence. The V3 score used as a measure of crystallized intelligence was computed by subtracting the number of incorrect answers divided by four from the total number of correct answers, in order to adjust for guessing.

\section{RESULTS}

To reduce the number of variables in a design with few degrees of freedom, $\mathrm{T} 1$ values for medial temporal gray and white matter were averaged across the hemispheres. Zero-order correlations among the cognitive, demographic, and radiological variables are presented in Table 1.

As hypothesized, the white-matter T1 exhibited a positive linear relationship with age. Because of Jernigan et al.'s (in press) finding of nonlinearity in age-T2 relationship, the data were examined for presence of higher order trends. The polynomial regression analysis revealed a significant quadratic trend that significantly increased the correlation between the white-matter $\mathrm{T} 1$ and age to $r=.63, p<.005$. The best-fit quadratic equation describing this relationship was: $\mathrm{T} 1_{w}=622.33-4.68 \cdot$ Age $+.066 \cdot$ Age $^{2}$.

The relationship between the gray-matter $\mathrm{T} 1$ and age was more complex. The linear correlation between the two variables was negative, as predicted, but small and statistically nonsignificant. However, the polynomial regression analysis revealed a significant cubic trend that increased the multiple correlation between the gray-matter $\mathrm{T} 1$ and the age components to $r=.54, p<.05$. The relationship between the hippocampal $\mathrm{T} 1$ and age was described by a cubic equation: $\mathrm{T}_{g}=394.98+20.98$ - Age - .528 $\cdot \mathrm{Age}^{2}+.004 \cdot \mathrm{Age}^{3}$. No correlation between gray- and white-matter T1 was found.

The percent ratio of gray- to white-matter $\mathrm{T} 1$ was linearly related to age, approximating $100 \%$ in the older subjects. As evident from the distribution in Figure 2a, the negative association between age and gray-whitematter differentiation index in the medial temporal lobes was not disproportionally influenced by outliers. Nonlinear regression analysis revealed no significant higher order trends.

Examination of relationships between the MRI parameters and cognitive performance confirmed that whitematter T1 was shorter (i.e., more "normal") in subjects who attained higher CFIT scores, whereas gray-matter $\mathrm{T} 1$ was unrelated to performance on that test. Unexpectedly, longer gray-matter T1 values were observed in subjects with lower vocabulary scores, although no relationship was found between those scores and the whitematter $\mathrm{T} 1$.

The gray-white-matter $\mathrm{T} 1$ ratio showed a significant linear relationship with both age and fluid intelligence (Ta-

Table 1

Means and Standard Deviations of the Variables of Interest and Zero-Order Correlations among Them

\begin{tabular}{lcccccc} 
& & & & \multicolumn{3}{c}{ T1 } \\
\cline { 5 - 7 } & CFIT & V3 & Education & Gray & White & Gray/White \\
\hline Age & $-.71 \ddagger$ & .30 & -.29 & -.25 & $.54 \dagger$ & $-.57 \dagger$ \\
CFIT & & .20 & $.57 \dagger$ & .19 & $-.56 \dagger$ & $.55 \dagger$ \\
V3 & & & $.56 \dagger$ & $-.44 *$ & .02 & -.28 \\
Education & & & & .08 & -.24 & .13 \\
Gray T1 & & & & & -.03 & $.55 \dagger$ \\
White T1 & & & & & & $-.84 \ddagger$ \\
Mean & 30.5 & 23.9 & 14.7 & 626.7 & 575.2 & 110.2 \\
SD & 6.1 & 11.2 & 2.8 & 44.5 & 60.7 & 14.5 \\
\hline
\end{tabular}

Note-CFIT $=$ Cattell Culture Fair Test. V3 = Extended Vocabulary. $\mathrm{T} 1=$ spin-lattice relaxation time (in milliseconds). Education in years. $\quad N=26 . \quad{ }^{*} p<.05 . \quad \dagger p<.005 . \quad \ddagger p<.001$. 


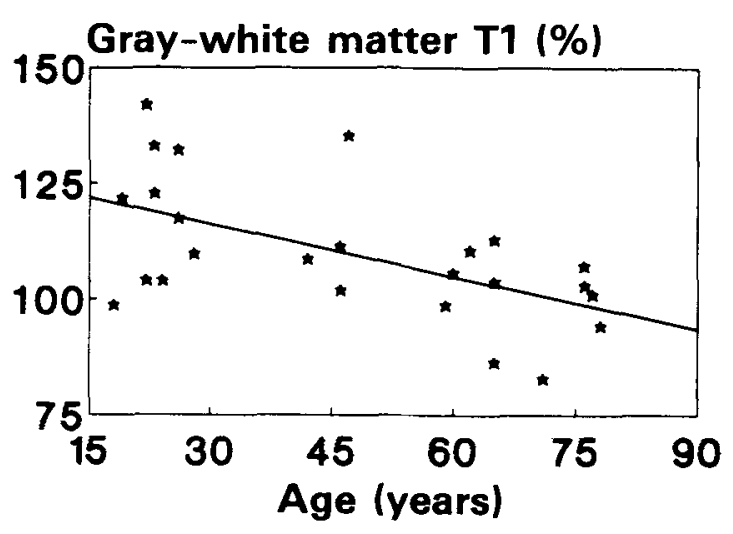

a.

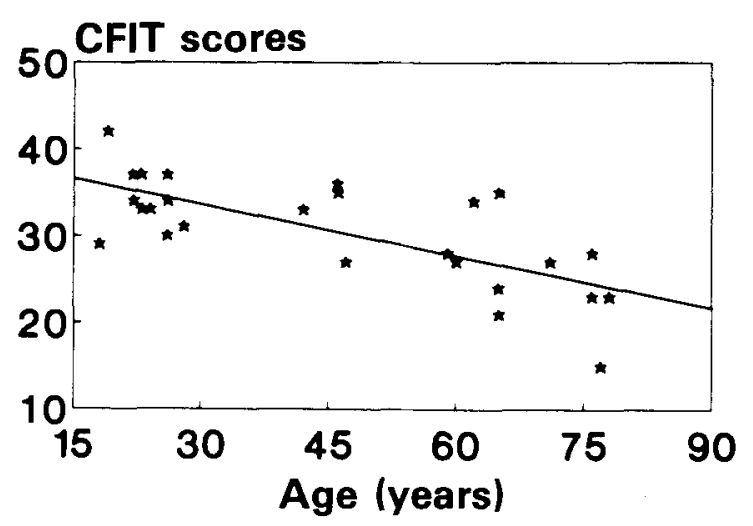

b.

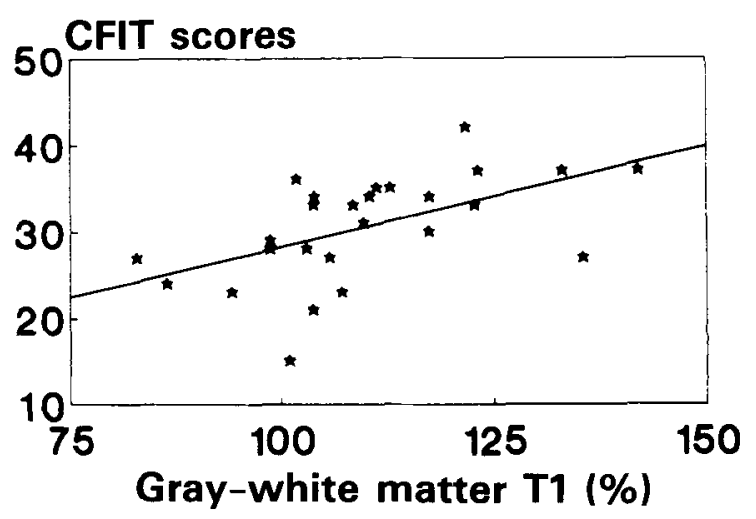

c.

Figure 2. Simple linear regression plots for the variables included in the multiple regression analysis: age, gray-white-matter $\mathrm{T} 1 \mathrm{ra-}$ tio, and CFIT scores.

ble 1). To partition the variance in CFIT and V3 scores into components attributable to age and to age-related changes in gray-white-matter differentiation, we conducted a hierarchical regression analysis. In this analysis, the independent variables (i.e., age and the gray-white $T 1$ ratio) were entered in two sets of linear models: one for fluid (CFIT), the other for crystallized (V3) intelligence. More than half of the variance in CFIT scores $\left(R^{2}\right.$ $\cdot 100 \%=53 \%$ ) was explained by the independent variables $[F(2,23)=12.94, p<.001]$, with $23 \%$ attributable to age alone $[F(1,23)=11.23, p<.001]$; about $3 \%$ was uniquely explained by the gray-white-matter $\mathrm{T} 1$ ratio $[F(1,23)=1.57$, n.s. $]$, with both variables accounting for $27 \%$ of the total variance in CFIT. In contrast, age and the $\mathrm{T} 1$ ratio did not explain a significant proportion of variance in $\mathrm{V} 3$ scores $\left[R^{2}=.10, F(2,23)=1.36\right.$, n.s.]. The multiple correlation between CFIT and the set of two independent variables-age and gray-white-matter $\mathrm{T} 1$ ratio-was significantly greater than that between $\mathrm{V} 3$ and the same pair of predictors: $Z^{*}=2.04, p<.05$ (Steiger, 1980).

\section{DISCUSSION}

The results of this study indicate that, at least in terms of molecular properties of tissue water, structural differences between gray and white matter of the medial temporal lobes diminish with age. Indeed, some elderly subjects show very poor differentiation between the two types of cerebral tissue. This finding provides a quantitative confirmation of George et al.'s (1981) qualitative observation of reduced gray-white-matter discriminability on CT scans of the elderly. It is also consistent with the reports of decreased gray-white-matter differentiation in the elderly based on rCBF and postmortem studies (Meyer, Kobari, Ichijo, Imai, \& Oravez, 1988; Samorajski \& Rolsten, 1973). Interestingly, the pattern of age-related decrease in gray-white-matter differentiation is the converse of the diverging pattern observed at the earliest stages of postnatal development (Barkovich, Kjos, Jackson, \& Norman, 1988; Holland, Haas, Norman, BrantZawadzki, \& Newton, 1986), with equality of gray- and white-matter T1 values found only in neonates (Bottomley et al., 1984). Although different processes may be responsible for reduced gray-white differentiation in the elderly, the resemblance of their final product illustrates how the development of the brain may have come full circle in the cycle of growth and decline.

The nonlinear age-related prolongation of the whitematter $\mathrm{T} 1$ found in this study is consistent with a similar quadratic trend for the white-matter T2 reported by Jernigan et al. (in press). The cubic age trend for hippocampal $\mathrm{T} 1$ suggests that whereas the gray-matter $\mathrm{T} 1$ in the elderly is shorter than in the young, in the "old" old T1 prolongation is observed. The reason fur this trend is unclear. One possibility is that cerebral atrophy resulting in enlargement of spaces filled with cerebrospinal fluid (CSF) may be the dominant process in the older elderly, whereas the brains of their younger peers may be more affected by age-related processes reducing the proportion of free water in the tissue. Indeed, comparison of graymatter $\mathrm{T} 1$ between the young (age $<35$ ) and the very old (age $>68$ ) subjects showed no differences [648.6 msec vs. $629.6 \mathrm{msec} ; t(14)=.79$, n.s.], whereas the younger elderly (age 60-68) exhibited a significant shortening of the hippocampal T1 $[600.6 \mathrm{msec} ; t(15)=$ $2.14, p<.05]$. Changes in temporal gray and white mat- 
ter frequently observed in the aging population may stem from a variety of loosely linked or even completely independent cerebral processes listed in the introduction to this report; a near-zero correlation between T1 times measured in cerebral white and gray matter is consistent with this view. Taken together, however, these measures may summarize the net result of multiple age-related cerebral changes in a single index.

It is unclear whether or not gray-white-matter dedifferentiation reflects a general trend in the aging brain and whether or not relationships between aging of specific brain structures and specific cognitive abilities may be established at all. This study provides evidence supporting an association between a general cognitive measure and tissue properties in a selected cerebral region. To assess the validity of this approach and its generality, future studies should include measurements of brain structures and cognitive abilities aimed at double dissociation of structure-function relationships. If the validity of $\mathrm{T} 1$ measures as indexes of the extent of age-related cerebral transformations is established, gray-white-matter discriminability may become a promising biological marker of cerebral aging. Provided the relationship between fluid intelligence and such a marker is replicated, we may obtain a powerful method of studying biological foundations of cognitive aging in vivo.

Sprott (1988) listed the following requirements for good biomarkers of aging: (1) be measurable in a noninvasive risk-free procedure, (2) reflect basic physiology and not pathology, (3) show broad generalizability across species, (4) be reproducible within and across laboratories, (5) have a measurable and predictable rate of change, and (6) show significant changes in relatively short periods of time. Evaluation of the proposed index of cerebral aging according to Sprott's criteria reveals that the gray-white ratio of $\mathrm{T} 1$ times completely satisfies only (1), while the rest of these criteria have yet to be met. We believe that careful selection of our sample makes meeting requirement (2) very likely. Requirement (3) is partially satisfied, for gray-white-matter $\mathrm{T} 1$ ratio in young laboratory mammals is similar to that measured in humans (Bottomley et al., 1984). We are not aware, however, of animal studies of age-related changes in gray-white-matter differentiation. Such studies are feasible and would be necessary for validation of gray-whitematter $\mathrm{T} 1$ ratio as a biomarker of aging. The question of satisfying (4) can be answered only by replication, whereas (5) and (6) require a longitudinal study. If these conditions are met, the gray-white-matter differentiation index will be useful in exploring the biological foundations of cognitive and emotional changes associated with old age, the response of the aging CNS to environmental insults, chronic medication use, and variations in nutrition.

In respect to cognitive correlates of cerebral aging, the results of this study are not clear-cut. A substantial agerelated difference in fluid intelligence was observed, as expected, and the zero-order correlation between CFIT scores and the index of gray-white-matter differentiation was moderately high. On the other hand, age alone accounted for a substantial proportion of variance in fluid intelligence, and the unique contribution of the white-gray-matter differentiation index was nonsignificant. It is important to emphasize, however, that the largest share of nonerror variance in fluid intelligence was explained by the commonality between age and the grayand white-matter properties. We could not find a readily ingerpretable association between $\mathrm{T} 1$ values in the hippocampal formation and age-related changes in cognition.

The correlations between the cognitive measures and the $T 1$ values were further examined in a subsample of elderly subjects (age $\geq 60$ years). When these subjects were considered separately, the direction and the magnitude of the correlations among age, CFIT, and the whitematter $\mathrm{T} 1$ were preserved. The older of the elderly subjects had longer white-matter $\mathrm{T} 1(r=.69, p<.01)$ and tended to exhibit lower CFIT scores $(r=.52, p<.1)$, whereas those with lower CFIT scores tended to have prolonged white-matter T1 $(r=.49, p<.13)$. The results of a similar analysis of the correlations among the gray-matter $T 1$, age, and vocabulary scores were quite different. Compared with the pattern observed in the full sample, the one revealed by the analysis of the subsample of the elderly was reversed: Gray-matter T1 tended to become longer, not shorter, with age $(r=.52, p<.1)$, and vocabulary scores showed a decreasing, not increasing, age-related trend $(r=-.51, p<.11)$; the correlation between V3 and gray-matter T1 dropped to $r=$ $-.20, p>.5$, n.s. Thus, a between-group comparison revealed that although the elderly subjects $(\geq 60$ years old) had shorter gray-matter $\mathrm{T} 1$ than did the younger subjects ( $\leq 35$ years old), they were also blessed with somewhat higher vocabulary scores. Within the elderly group, however, the oldest subjects showed age-related prolongation of the T1 times and a decline in verbal ability. These analyses suggest that the unexpected negative correlation between verbal ability and hippocampal T1 observed in the total sample is, probably, an artifact. The predicted positive relationship between CFIT scores and hippocampal T1 did not materialize.

The reported findings, although preliminary in nature, raise several important questions for future research. Calendar age is not a specific variable; it represents a variety of physiological and cognitive factors that may influence performance on complex tasks, such as fluid intelligence tests. We cannot, therefore, adopt a model postulating that age affects fluid intelligence directly and indirectly via the measured brain variable ( $\mathrm{T} 1$ ratio). The problem with such a model is that the relationship between age and a single cerebral measure is not unidirectional. Although the calendar age is obviously not influenced by cerebral integrity, the physiological variables that it represents may be. Thus, the appropriate model would be one including age and gray-white $\mathrm{T} 1$ ratio as correlated causes of decline in CFIT. Therefore, in spite of the fact that a substantial proportion of variance is explained by age alone, it is the commonality between the measured 
brain variable and those contained under the "age" label that may draw the interest of future investigators. Unlike an interaction indicating joint effect of the independent variables on the dependent one, commonality suggests redundancy of the influence of the independent variable on the dependent. Further partitioning of the variance hidden in this common part is a challenging task that will require exploration of additional indexes of neural aging within a framework of multivariate study. Such indexes would include electrophysiological and metabolic measures of brain work, as well as performance on elementary information-processing tasks representing fundamental components of intelligence.

\section{REFERENCES}

ANSARI, K. A., \& LOCH, J. (1975). Decreased myelin basic protein content of the aged human brain. Neurology, 25, 1045-1050.

Barkovich, A. J., KJos, B. O., Jackson, D. E., Norman, D. (1988). Normal maturation of the neonatal and infant brain, MR imaging at 1.5 T. Radiology, 166, 173-180.

Barnes, C. A. (1983). The physiology of the senescent hippocampus. In W. Seifert (Ed.), Neurobiology of the hippocampus (pp. 87-108). London: Academic Press.

Bottomley, P. A., Foster, T. H., Argersinger, R. E., Pfeifer, L. M. (1984). Review of normal tissue hydrogen NMR relaxation times and relaxation mechanisms from 1-100 MHz: Dependence on tissue type, NMR frequency, temperature, species, excision, and age. Medical Physics, 11, 425-448.

Bourne, G. H. (1973). Lipofuscin. In D. H. Ford (Ed.), Neurobiological aspects of maturation and aging. Amsterdam: Elsevier.

CATtell, R. B., CATTELl, A. K. S. (1973). Handbook for the individual or group culture-fair inielligence tes: Scale 2. Champaign, IL: IPAT.

Coleman, P. D., \&Lood, D. G. (1987). Neuron numbers and dendritic extent in normal aging and Alzheimer's disease. Neurobiology of Aging, 8, 521-545.

Daniels, D. L., Naughton, V. M., Naidich, T. P. (1987). Cranial and spinal MRI: An atlas and guide. New York: Raven Press.

de Leon, M. J., George, A. E., Stylopoulos, L. A., Smith, G., \& MiLLER, D. C. (1989). Early markers for Alzheimer's disease: The atrophic hippocampus. Lancet, II, 672-673.

Ekstrom, R. B., French, J. W., Harman, H. H., Derman, D. (1976). Manual for kit of factor-referenced cognitive tests. Princeton, NJ: Educational Testing Services.

Folstein, M. F., Folstein, S. E., \& MCHugh, P. R. (1975). Minimental state: A practical method for grading the cognitive state of patients for the clinician. Joumal of Psychiatric Research, 12, 189-198.

George, A. E., de Leon, M. J., Ferris, S., \& Kricheff, I. I. (1981). Parenchymal CT correlates of senile dementia (Alzheimer disease): Loss of gray-white matter discriminability. American Joumal of Neuroradiology, 2, 205-213.

Ginsberg, M. D., Hedley-Whyte, T., \& Richardson, E. P. (1975). Hypoxic-ischemic leukoencephalopathy in man. Archives of Neurology, 33, 5-14.

HARMS, S. D., \& KRAMER, D. M. (1985). Fundamentals of magnetic resonance imaging. CRC Critical Reviews in Diagnostic Imaging, 25, 79-111.

Holland, B. A., Haas, D. K., Norman, D., Brant-Zawadzk, M., \& NEWTON, T. H. (1986). MRI of normal brain maturation. American Journal of Neuroradiology, 7, 201-208.

HORN, J. L. (1986). Intellectual ability concepts. In R. J. Sternberg (Ed.), Advances in psychology of human intelligence. Hillsdale, NJ: Erlbaum.

Jernigan, T. L., Archibald, S. L., Berhow, M. T., Sowell, E. R., Foster, D. S., \& Hesselink, J. R. (in press). Cerebral structure on MRI: 1. Localization of age-related changes. Biological Psychiatry. Kirkpatrick, J. B., Hayman, L. A. (1987). White-matter lesions in MR imaging of clinically healthy brains of elderly subjects: Possible pathological basis. Radiology, 162, 509-511.

Leys, D., Soetaerst, G., Petit, H., Faukette, A., Pruvo, J. P., * Steinling, M. (1990). Periventricular and white matter magnetic resonance imaging hyperintensities do not differ between Alzheimer's disease and normal aging. Archives of Neurology, 47, 524-527.

MacDonald, H. L., Bell, B. A., Smith, M. A., Kean, D. M., Douglas, R. H. B., Tocher, J. L., MiLler, J. D., a BeST, J. J. K. (1985). In vivo correlation of NMR T1 and brain water. British Joumal of Radiology, 58, 817.

Mani, R. B., Lohr, J. B., Jeste, D. V. (1986). Hippocampal pyramidal cells in the human: A quantitative study of neuronal loss in sectors CA1 through CA4. Experimental Neurology, 94, 29-40.

MATHUR-DeVRÉ, R. (1984). Biomedical implications of the relaxation behavior of water related to NMR imaging. British Joumal of Radiology, 57, 955-976.

Meyer, J. S., Kobari, M., Ichuo, M., Lma, A., Oravez, W. (1988). Solubility of Xenon gas in human brain increases during normal aging: Pathological implications. Annals of Neurology, 21, 133.

Morone, T., Benevento, J., Di Massimo, R., Martino, A., ORBACH, E., WEIss, M. (1987). In vivo Nuclear Magnetic Resonance spin-lattice relaxation time measurement from restricted volumes. American Journal of Physiological Imaging, 2, 17-23.

Press, G. A., Amaral, D. G., Squire, L. R. (1989). Hippocampal abnormalities in amnesic patients revealed by high-resolution magnetic resonance imaging. Nature, 341, 54-57.

SAMORAJSKI, T., Rolsten, C. (1973). Age and regional differences in chemical composition of brains of mice, monkeys, and humans. In D. H. Ford (Ed.), Neurobiological aspects of maturation and aging. Amsterdam: Elsevier.

SprotT, R. L. (1988). Age-related variability. Annals of New York Academy of Sciences, 515, 121-123.

SQuIRE, L. R. (1986). Mechanisms of memory. Science, 232, 1612-1619.

STEIGER, J. H. (1980). Tests for comparing elements of a correlation matrix. Psychological Bulletin, 87, 245-251.

Sze, G., DE ARmond, S. J., Brant-Zawadzki, M. Davis, R. L., Norman, D., * Newton, T. H. (1986). Foci of MRI signal (pseudo lesions) anterior to the frontal horns: Histologic correlations of a normal finding. American Joumal of Neuroradiology, 7, 381-387.

Terry, R. D., DeTeresa, R., Hansen, L. A. (1987). Neocortical cell counts in normal human adult aging. Annals of Neurology, 21, 530-539.

Tomlinson, B., Blessed, G., \& Roth, M. (1968). Observations on the brains of non-demented old people. Joumal of Neurological Sciences, 7, 331-356.

Tomonaga, M., Yamanouchi, H., Tohgi, H., \& Kameyama, M. (1982). Clinicopathological study of progressive subcortical vascular encephalopathy (Binswanger type) in the elderly. Joumal of American Geriatric Society, 30, 524-529.

Unger, E., LitTlefield, J., \& GADO, M. (1988). Water content and water structure in CT and MR signals: Possible influence in detection of early stroke. American Joumal of Neuroradiology, 9, 687-691.

Wahlund, L.-O., Agartz, I., AlmQvist, O., Basun, H., ForsSELL, L., SAAF, J., WeTterberg, L. (1990). The brain in healthy aged individuals: MR imaging. Radiology, 174, 675-679.

White, L. R., Cartwright, W. S., Cornoni-Huntley, J., Brock, D. B. (1986). Geriatric epidemiology. Annual Review of Gerontology and Geriatrics, 6, 215-311.

Wiggins, R. C., Gorman, A., Rolsten, C., SAmorajski, T., BalLINGER, W. E., FreUND, G. (1988). Effects of aging and alcohol on the biochemical composition of histologically normal human brain. Metabolic Brain Diseases, 3, 67-80.

Winocur, G. (1988). A neuropsychological analysis of memory loss with age. Neurobiology of Aging, 9, 487-494.

WISNIEWSKI, H. M., TERRY, R. D. (1973). Morphology of the aging brain, human and animal. In D. H. Ford (Ed.), Neurobiological aspects of maturation and aging. Amsterdam: Elsevier.

(Manuscript received January 22, 1990; revision accepted for publication August 29, 1990.) 